\title{
Advanced Loss Eventuality Assessment and Technical Estimates: An Inte- grated Approach for Management of Healthcare-Associated Infections
}

\author{
Vittorio Gatto $^{1 \#}$, Matteo Scopetti ${ }^{1 \#,}$ Raffaele La Russa ${ }^{1,2^{*}}$, Alessandro Santurro ${ }^{1}$, Luigi Cipolloni ${ }^{1}$, \\ Rocco Valerio Viola ${ }^{1}$, Mariantonia Di Sanzo ${ }^{1}$, Paola Frati ${ }^{1,2}$ and Vittorio Fineschi ${ }^{1,2}$ \\ ${ }^{I}$ Department of Anatomical, Histological, Forensic and Orthopaedic Sciences, Sapienza University of Rome, Rome, Italy; \\ ${ }^{2}$ IRCCS Neuromed, Pozzilli, Italy
}

A R T I C L E H I S T O R Y

Received: November 20, 2018 Revised: December 15, 2018 Accepted: January 02,2019

DOI:

$10.2174 / 1389201020666190408095050$
Abstract: Background: Healthcare Associated Infections (HAIs) represent a crucial issue in health and
patient safety management due to the persistent nature, economic impact and possible preventability of
the phenomenon. Compensation claims for damages resulting from HAI could provide insights that can
improve the understanding of suboptimal steps in the therapeutic process, enable an estimate of costs re-
lated to infectious complications, and guide the development of planning tools for implementation of the quality of care.

Objective: This paper analyzes all the HAI claims received at the Umberto I General Hospital of Rome across a five-year period with the aim of outlining a methodological approach to the litigation management and of characterizing the economic impact of infections on health facilities resources.

Method: All claims received during the study period have been classified according to the International Classification for Patient Safety (ICPS) system. Subsequently, claims related to Healthcare Associated Infections were evaluated through an innovative tool for determination of the risk of loss, the Advanced Loss Eventuality Assessment (ALEA) score.

Results: The results obtained demonstrate the relevance of a correct management of HAI claims in the administration of a health care system. Specifically, the cases examined during the study highlighted the significant impact of infectious diseases of a nosocomial nature in terms of frequency and economic exposure.

Conclusion: The proposed methodological approach allows a productive analysis of the internal processes, providing fundamental data for the refinement of the preventive strategies and for the rationalization of the resources through the expenditure forecasts.

Article Highlights Box: Healthcare-Associated Infections represent an essential element to consider in the management of health facilities.

- Many studies highlight the economic burden of Healthcare-Associated Infections in health policies.

- Litigation management represents a useful resource in the prevention of Healthcare Associated Infections.

- Appropriate clinical risk management policies in the field of Healthcare-Associated Infections allow the implementation of preventive measures, the reduction of the incidence of the phenomenon and the quality of care.

- The costs of Healthcare-Associated Infections can be limited through a systematic methodological approach based on Advanced Loss Eventuality Assessment and technical estimate of the value of each case.

- The application of a standardized system would be desirable in any health facility despite the potential methodological, technical, behavioral and financial issues.

Keywords: Healthcare-Associated Infections, litigation management, HAI claims, ALEA score, technical estimates, cost analysis, quality of health care, patient safety.

\footnotetext{
*Address correspondence to this author at the Department of Anatomical, Histological, Forensic and Orthopaedic Sciences, Sapienza University of Rome, Viale Regina Elena 336, 00185 Rome, Italy; Tel: +390649912722; Fax: +390649912097; E-mail: raffaele.larussa@uniroma1.it

${ }^{\#}$ Equal contribution.
}

\section{INTRODUCTION}

Around the world, health systems face a wide range of challenges such as the imperative need to improve the quali- 
ty and safety of care, the goal of facilitating access and the obligation to manage increasing healthcare costs [1-4].

Healthcare-Associated Infections represent the most frequent adverse events during hospitalization and constitute an important health problem due to prolonged hospital stays, increased long-term disability and death, selection of antibiotic-resistant microorganisms. and the financial burden on health systems $[5,6]$. According to the definition developed by the World Health Organization, a HAI can be described as "An infection occurring in a patient during the process of care in a hospital or other health-care facility which was not present or incubating at the time of admission. This includes infections acquired in the hospital, but appearing after discharge, and also occupational infections among the staff of the facility" [7].

Although HAIs represent a frequent adverse event in the health sector, the true incidence of the phenomenon at a global level remains unknown because in most countries there are no surveillance systems for nosocomial infections [8]. According to the European Centre for Disease Prevention and Control (ECDC), over 3.2 million patients throughout the European Union have at least one HAI following hospitalization with a total of 37,000 deaths as a direct consequence of infection $[9,10]$.

An increasing amount of evidence is demonstrating that HAIs represent a unique opportunity to save lives and rationalize costs for all professionals involved in implementing the quality of care [11-13]. In particular, various scientific contributions make assessments on the cost-effectiveness of a wide variety of interventions for the prevention and control of nosocomial infections. However, many studies provide evidence of poor quality and hardly applicable to different health systems. For these reasons, a standardization process of the definitions and the operating procedures appears necessary to make the prevention of Healthcare-Associated Infections achievable even in contexts where currently the resources are limited.

Compensation claims for damages resulting from HAI provide insights that improve the understanding of suboptimal steps in the therapeutic process, enable an estimate of costs related to infectious complications, and guide the development of planning tools for the amelioration of care safety [14-18].

The present study is based on the analysis of litigation of a high-income hospital such as the Umberto I general hospital in Rome. In particular, the authors carried out a study on HAI claims with the aim of outlining a methodological approach to the litigation management and of characterizing the economic impact of infections on health facilities resources [19].

The proposed approach is based on an integrated evaluation of HAI claims by determining the risk of loss and technical estimates. Regarding the first task, it has been created a scoring system based on objective parameters evaluable from the medico-legal point of view, the Advanced Loss Eventuality Assessment (ALEA). For the determination of the technical estimates, it has instead adopted a systematic procedure based on common evaluation systems of personal impair- ment in order to quantify the claim reserves on current liabilities for claims reported but not yet settled.

\section{MATERIALS AND METHODS}

The study was conducted through the analysis of data on the activity carried out by the Claims Assessment Committee of Umberto I General Hospital in Rome from January 2013 to December 2017. All claims received during the study period have been classified according to the (ICPS) system by incident type and patient outcome. Subsequently, claims related to Healthcare Associated Infections were selected and a medico-legal assessment was carried out to identify the inadequacies of the care path.

The risk of loss was determined using the Advanced Loss Eventuality Assessment (ALEA) score, an evaluation tool designed specifically for HAI (Fig. 1). This scoring system is based on the analysis of 14 items represented by:

- Annual definition of objectives for risk management (score 1);

- $\quad$ Procedures for reporting and managing near misses, sentinel events and adverse events (score 1);

- Procedures for notification of detection of alert organisms (score 1);

- $\quad$ Procedures for environmental disinfection (score 1);

- Guidelines for hospital isolation measures (score 1);

- Guidelines for the correct use of antiseptics and disinfectants (score 1);

- Guidelines for perioperative antibiotic prophylaxis (score 1);

- Additional procedures for the prevention of HAI relevant to the specific case (score 1);

- Quality of the medical record (score 2);

- Asepsis indicators (score 2);

- Antibiotic therapy (score 2);

- Infectivological consulting (score 2);

- Control, surveillance, and prevention activities through the HAI Committee (score 2);

- Demonstration of the application of adequate preventive measures in the specific case (score 5).

For each of the items, failure to satisfy the requirement results in a score of 0 . Loss eventuality was stratified according to the score obtained in three risk categories consisting of high risk (score $<12$ ), medium risk (score 12-18) and low risk (score $>18)$

A further analysis was performed in order to determine a technical estimate of the value of each case (Fig. 2). This task was carried out through a preliminary distinction between cases in which there were permanent impairment and cases in which death occurred. In cases of permanent impairment, the value of the claim has been quantified on the basis of a tabular system for the evaluation of the outcomes. In cases of damage from parental loss, the estimate was instead determined through an algorithm based on the age of 


\section{Advanced Loss Eventuality Assessment (ALEA)}

\begin{tabular}{|c|c|c|}
\hline Item & Inadequate & Adequate \\
\hline Annual definition of objectives for risk management & 0 & 1 \\
\hline Quality of the medical record & 0 & 2 \\
\hline Asepsis indicators & 0 & 2 \\
\hline Appropriateness antibiotic therapy & 0 & 2 \\
\hline Infectivological consulting & 0 & 2 \\
\hline Procedures for reporting and managing near misses, sentinel events and adverse events & 0 & 1 \\
\hline Procedures for notification of detection of alert organisms & 0 & 1 \\
\hline Procedures for enviromental disinfection & 0 & 1 \\
\hline Guidelines for hospital isolation measures & 0 & 1 \\
\hline Guidelines for the correct use of antiseptics and disinfectants & 0 & 1 \\
\hline Guidelines for perioperative antibiotic prophylaxis & 0 & 1 \\
\hline Additional procedures for the prevention of Healthcare Associated Infections relevant to the specific case & 0 & 1 \\
\hline Control, surveillance and prevention activities through the Committee for Healthcare Associated Infections & 0 & 2 \\
\hline Demonstration of the application of adequate preventive measures in the specific case & 0 & 5 \\
\hline $\begin{array}{c}\text { Medium risk } \\
12-18\end{array}$ & $\begin{array}{c}\text { Low risk } \\
19-23\end{array}$ & \\
\hline
\end{tabular}

Fig. (1). Advanced loss eventuality assessment score.

the victim, on the age of the relative, the degree of kinship and cohabitation with the victim.

\section{RESULTS}

The Umberto I general hospital counts on 1,200 beds for a total of 38,000 annual hospitalizations, 27,000 Day Hospital admissions and 2,303,046 outpatient accesses.

The study involved the analysis of 686 claims reported from January 2013 to December 2017.

With reference to the general claims, HAI claims showed an increase both in absolute and relative terms, from 12 of $167(7.2 \%)$ in 2013 to 16 of $124(12.9 \%)$ in 2017 (Fig. 3).

Following the classification by incident type and patient outcome according to the ICPS system, it was possible to identify 71 cases $(10.3 \%)$ of Healthcare Associated Infection with 39 cases $(55 \%)$ in which death occurred as a direct consequence of infection. In patients where the infection did not result in death, permanent impairment was absent in 2 cases $(2.8 \%)$, mild in $10(14.1 \%)$, moderate in $15(21.1 \%)$ and severe in 5 (7\%) (Fig. 4).
The evaluation of the latency time between the date of the event and the date of the complaint (Fig. 5a) showed a mean value of 34 months (range 1-120), highlighting a relative stability during the study period. Regarding this aspect, it was also noted that 36 claims $(50.7 \%)$ were reported within 24 months from the date of the adverse event, while all the remaining complaints occurred within the remaining 96 months required by law.

The evaluation of the risk of loss through the Advanced Loss Eventuality Assessment score allowed to establish the prevalence of claims at high risk $(44 ; 62 \%)$ followed by claims at low $(16 ; 22.5 \%)$ and medium $(11 ; 15.5 \%)$ risk. The cross-analysis of the data obtained through the application of the ICPS system and the ALEA score has made it possible to highlight the prevalence of fatal claims $(28 ; 63.6 \%)$ in the category with a high risk of loss.

The determination of technical estimates (Fig. 5b) demonstrated the high potential economic impact of the claims analyzed allowing to quantify a forecast of expenditure of $31,062,500$ euro. Specifically, the mean value of disputes was 437,500 euro (range 0,00-1,500,000) with 34 claims $(47.9 \%)$ whose estimated value exceeded 500,000 euro. 


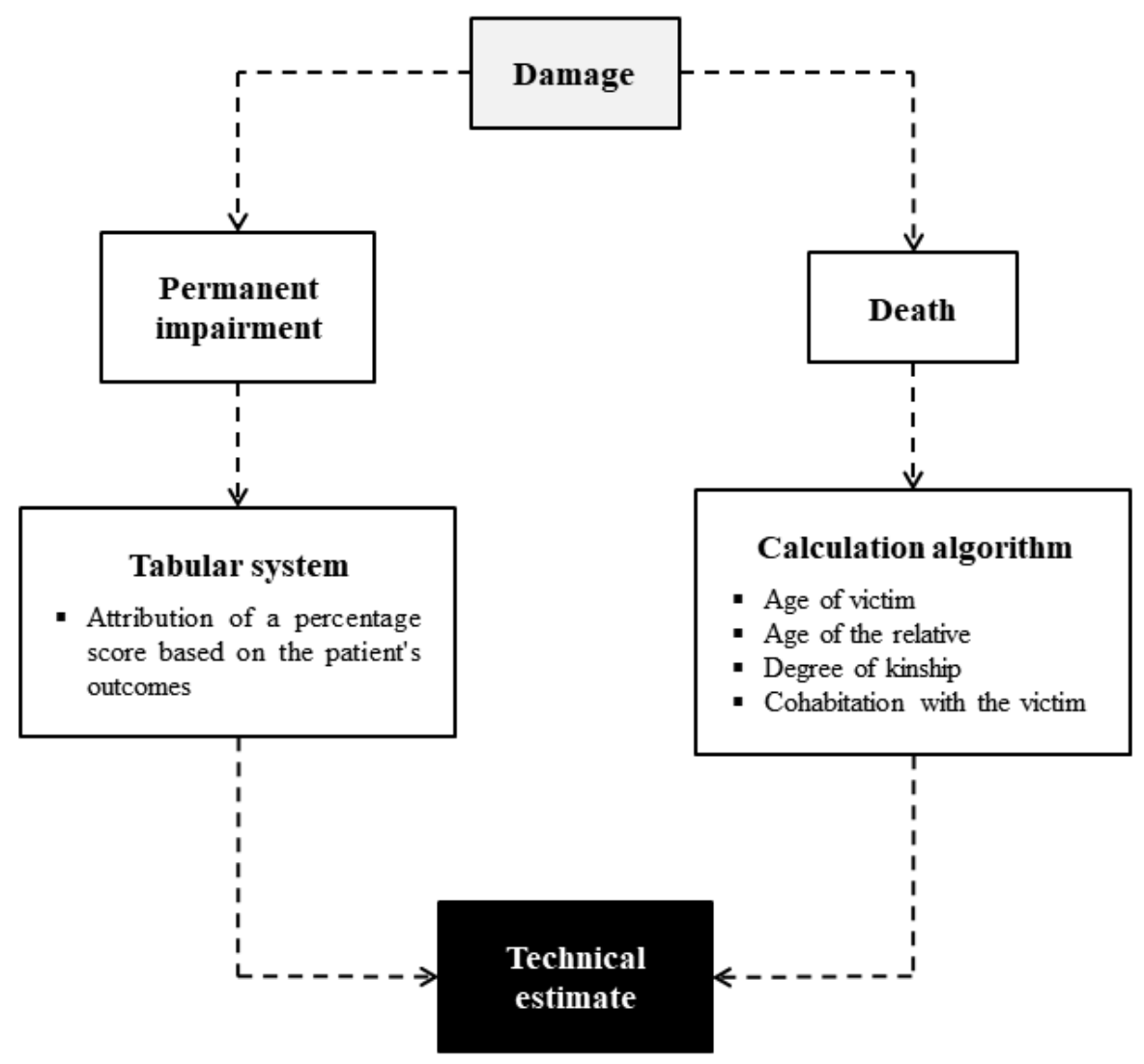

Fig. (2). Method for determining the technical estimate.

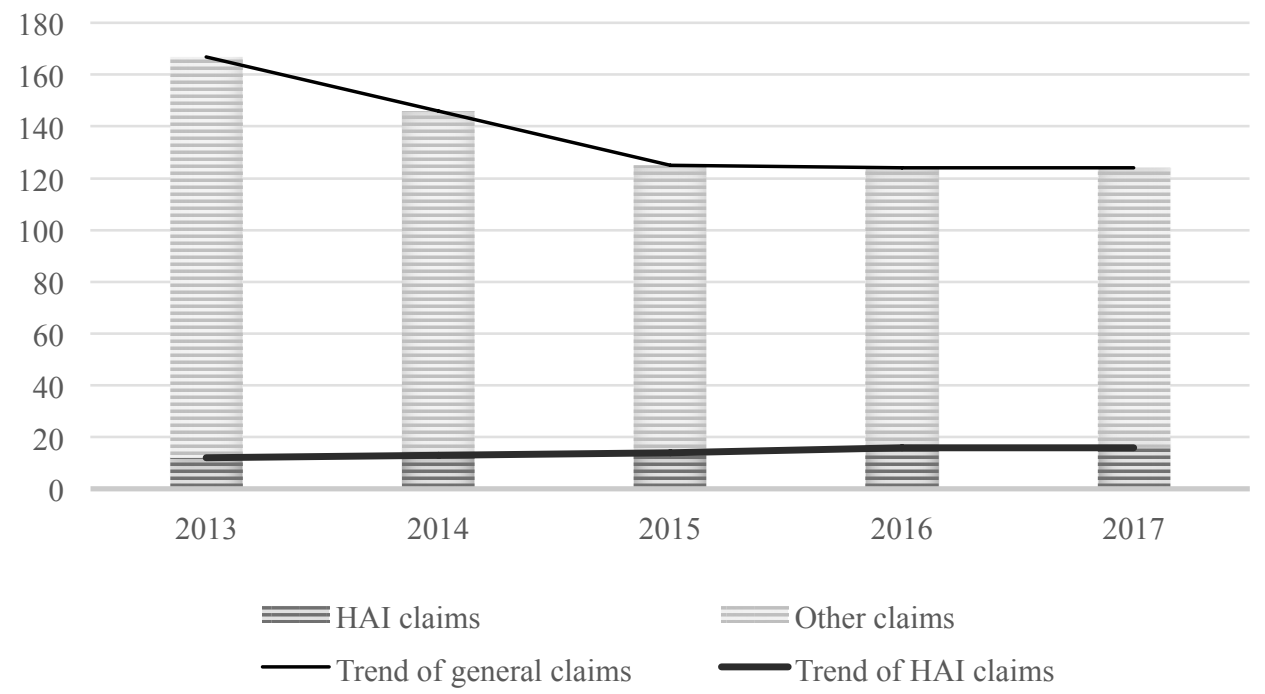

Fig. (3). Distribution of HAI claims with respect to other claims.

\section{DISCUSSION}

Healthcare Associated Infections (HAIs) are a crucial issue in health and patient safety management due to the persistent nature, economic impact and possible preventability of the phenomenon [20-22]. Specifically, nosocomial infections are still a major problem in health policies around the world due to the high impact on health systems not only in economic terms [23].
Several studies conducted on the topic have univocally demonstrated the benefits of reducing the number of nosocomial infections as well as the need for common efforts by health professionals for the prevention and control of HAIs $[24,25]$.

This study aims to highlight the importance of a conscious and scientific working method that allows to identify critical issues of the care system and to prevent nosocomial 
10

8

6

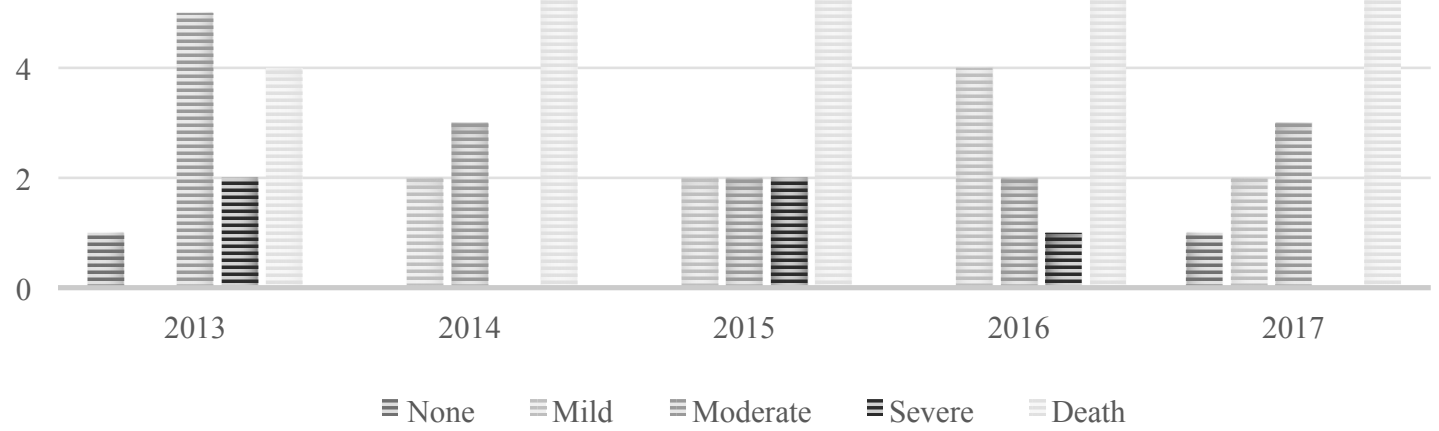

Fig. (4). Classification of HAI claims according to patient outcome.

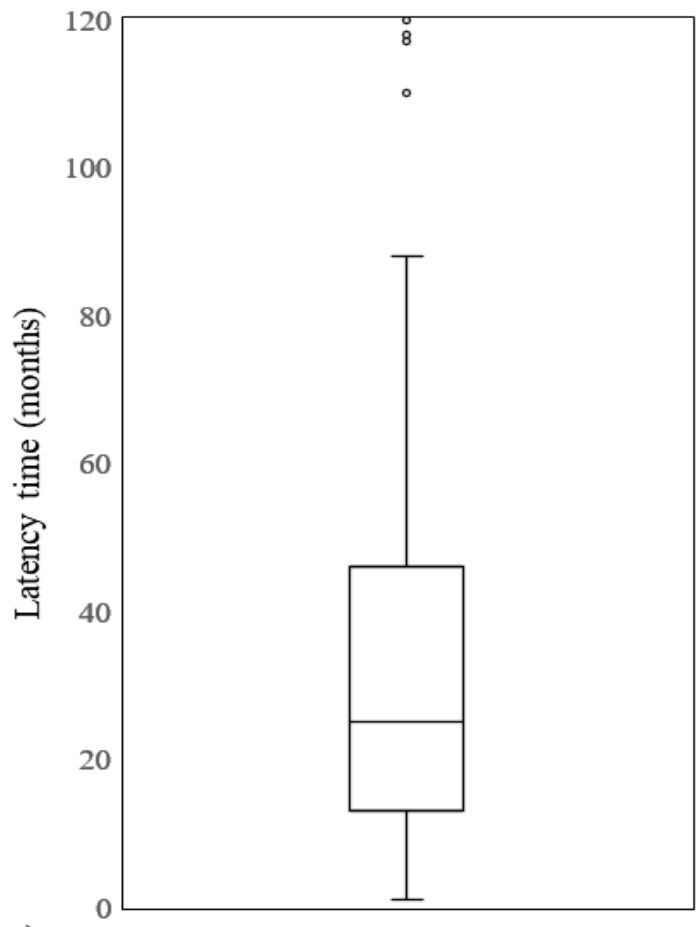

a)

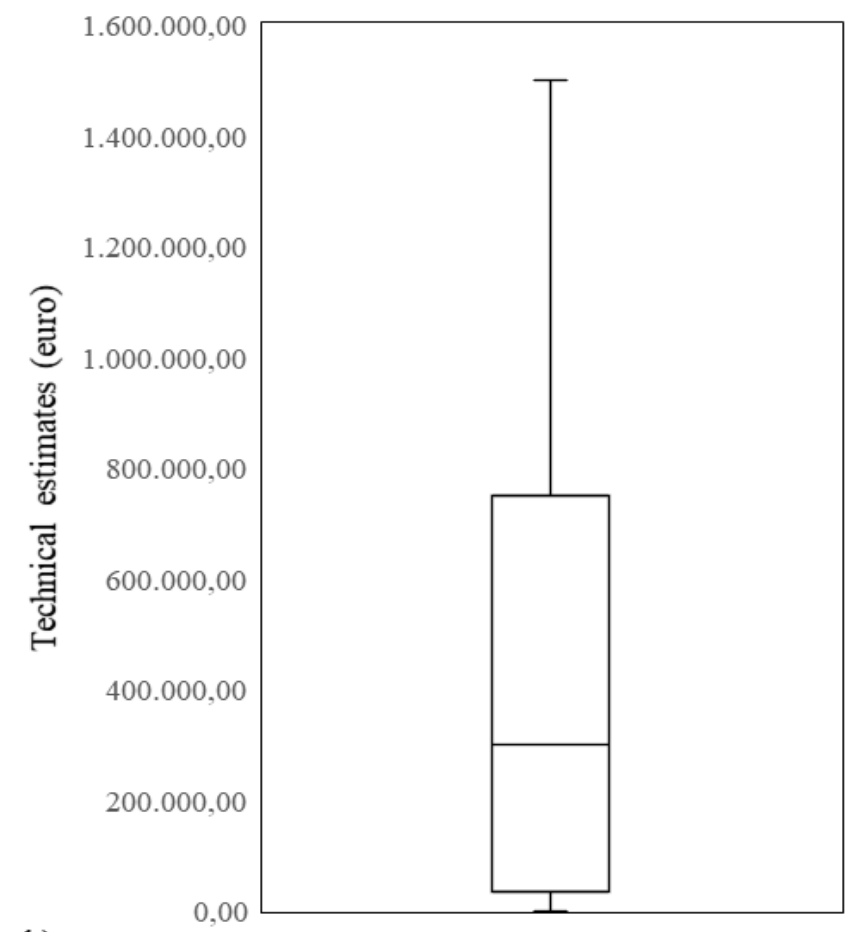

b)

Fig. (5). a) Latency time between the date of the event and the date of the complaint. b) Distribution of technical estimates.

infections starting from litigation data. In this sense, the results obtained showed that ALEA score and technical estimates represent particularly useful tools in the management of HAI claims.

With regard to the trend in claims during the study period, a decrease in the overall claims rate was recorded against an increase - in absolute and relative terms - of HAI claims. This can be justified on the basis of the growing expectations of the population on the levels of care and on the belief that it is unacceptable to contract an infection in a hospital environment.

Results related to patient outcomes demonstrate the high weight in terms of morbidity and mortality of nosocomial infections [26-32]. This evidence is even more significant because in the present study the evaluation of outcomes was carried out, as from the medico-legal method, after a long 
time from the acute event, with the clinical recovery from disease.

The analysis of the 71 HAI claims occurred during the study period allowed to establish that additional costs related to the management of nosocomial infections are to be ascribed, even before the payment of compensation, to the lengthening of hospital stay, use of antibiotics, additional procedures and interventions, re-admissions and postdischarge health care. In agreement with the findings, numerous international studies have demonstrated the high economic impact of nosocomial infections on healthcare systems [33, 34]. Specifically, a meta-analysis of costs and the financial impact on the US Health Care System has shown that the total annual costs for the main types of infections related to assistance are about 9.8 billion dollars, with a greater contribution to the determination of the overall costs of surgical site infections, ventilator-associated pneumonia, and central line-associated bloodstream infections $[35,36]$. A similar survey conducted by the European Center for Disease Prevention and Control has shown that total expenditure for nosocomial infections in the European Union can be estimated at around 7 billion euro a year, considering only the costs directly related to health care and not indirect costs attributable to damages of pecuniary and non-pecuniary nature [37]. As regards the rate of deaths associated with nosocomial infections, some studies conducted mainly in highincome countries showed mortality ranging from $12 \%$ to $80 \%$ depending on the population studied [38]. In a prevalence study conducted in 17 countries of the European Union, it was found that patients with nosocomial infections have higher mortality than patients who are not affected with an independent correlation between infectious complications (sepsis, pneumonia and bloodstream infections) and increased risk of death in critically ill patients [39]. Several other studies have also documented the different impact of the various types of Healthcare Associated Infection on mortality rates, for example by documenting that Ventilator Associated Pneumonia (VAP) has an estimated mortality between 7 and $30 \%$ and costs of about 10,000-25,000 dollars for each case [40,41]. Similarly, in the intensive care units of US hospitals, death occurred in approximately 28,000 of the 250,000 cases of Catheter-Related Bloodstream Infections (CR-BSI) annually recorded with costs estimated at around 2.3 billion dollars [42].

The data deriving from the determination of the risk of loss and technical estimates have shown, albeit in relation to the experience of a single health facility, the importance of the costs related to compensation for damage from nosocomial infections in the context of healthcare expenditure [43]. Managing the risk of loss related to HAI claims represents a major issue within healthcare facilities [44]. This task presents several challenges in terms of reporting and provision of economic resources due to the complexity of the risk management systems used and the difficulties in adopting standardized approaches [45-48]. Accuracy in estimating claims related to nosocomial infections is essential for hospitals in order to provide for the determination of expenditure forecasts and to implement risk prevention strategies [4952]. However, while in some cases it is possible to precisely quantify the predictable costs of compensation, in other situations this analysis can be extremely difficult due to the complexity of the care path or the type of damage that has occurred. For these reasons, the availability of appropriate data and information is essential for an accurate estimate of unpaid claims.

The results concerning the risk of loss suggests numerous insights about the current trends in nosocomial infections. The large proportion of claims at high risk of loss leads to significant implications for the costs of compensation for medical liability and the premiums to be insured against these costs in facilities where the risk retention is transferred to an insurance company. The rationale behind the incidence of HAI claims at high risk of losing lies basically in the difficulty in proving the effective application of the necessary preventive measures. Another problem in the management of HAI requests is the difficulty for patients, but especially for jurisprudence, to conceive of the possibility that infections can be contracted even in a hospital context. In view of the above, it is therefore also very difficult to obtain proof that the reported infection is part of the nosocomial infections that are not preventable. For all these reasons, in the field of HAI claims, it is essential to demonstrate the sensitivity of the health facilities to the issue of nosocomial infections by putting together, as a corollary of medico-legal evaluation on the case, all the documentation concerning the prevalence surveys conducted in the wards and the preventive procedures in use inside the structure [53].

The overall evaluation of HAI claims at the Umberto I general hospital also offers many indications about the litigation management dynamics. In particular, the data concerning the latency times between the event and the complaint show a close proximity between the two moments with about $50 \%$ of the claims that are reported within 24 months of the adverse event. In addition to reflecting the sensitivity of the population to the issue of Healthcare-Associated Infections, this finding highlights the need for the structures to proceed to a rapid and accurate definition of the case both as regards the preliminary phase and the determination of the risk of losing and technical estimates. This last aspect assumes considerable importance in view of the relief of succumbing possibility and dispute value in the definition of the subsequent defensive strategies.

On the basis of the foregoing, it is clear that the adoption of an integrated system for management of litigation and clinical risk deriving from Healthcare Associated Infections presupposes the employment of highly specialized and multidisciplinary personnel $[54,55]$.

Given the difficulty of implementing general systems for surveillance, control, and prevention of nosocomial infections in reality as the Italian one in which health policies are decentralized, claims are a useful tool for an analysis of the events concerning patient safety during the care path. However, the use of malpractice claims data, although proven to be effective, is not useful in reducing the rate of nosocomial infections if not corroborated by the implementation of surveillance and control measures [56, 57].

The surveillance of nosocomial infections is a complex task that requires case research through the joint work of clinicians and control teams, the definition of cases in which there is no microbiological diagnosis, the acquisition of all 
additional information for the determination of sources of infection and prevalence of HAI [58-62]. The difficulties in monitoring and controlling nosocomial infections lie in the need for a particular experience in the collection of epidemiological data, but above all in the evaluation of quality and in the interpretation of the same for the planning of interventions to adapt existing prevention measures [63]. In the European context, the first surveillance systems have been established since the beginning of the $90 \mathrm{~s}$ on the basis of the proven effectiveness of the US model based on the National Nosocomial Infection Surveillance Scheme of the Centers for Disease Control (CDC) [64]. In consideration of the different needs of infection control in the different EU countries, over the years several surveillance protocols have been developed. However, given the need to standardize surveillance systems, Europe has funded the HELICS project (Hospitals in Europe Link for Infection Control through Surveillance) since 2000 [65, 66]. However, although hospital-wide surveillance of all types of infections is of extreme epidemiological interest, general monitoring is rarely applicable as it currently does not allow useful comparisons between different realities and implies an excessive workload for staff involved in infection monitoring. Therefore, litigation management can provide a valid contribution to the surveillance systems, despite being an approach based on the analysis of events that have already taken place $[67,68]$. In fact, HAI claims are able to provide complete data on all types of infections, even if on a small scale, in consideration of the fact that not all nosocomial infections are reported for damages compensation. This study demonstrates the effective possibility of establishing a virtuous mechanism for the analysis of internal processes and self-education as well as for the reduction of HAI claims through the combination of infection control and surveillance systems. The results obtained should be interpreted by nosocomial infection control professionals as a reflective point of view for the creation of prospective surveillance systems and for the implementation of measures to reduce the exposure of health facilities in the event of litigation.

The present study has its main limit in the unavailability of data on out-of-court or judicial outcomes of the analyzed claims. This circumstance prevents the formulation of considerations regarding the long-term effectiveness of the proposed approach. For this reason, further studies are considered necessary for the validation of applicability and effectiveness of a litigation management system based on the systematic determination of the risk of loss and technical estimates. Reliable results on the analyzed cases can be obtained at the close of the analyzed claims through the information regarding the judicial outcomes and the amounts settled. At present, prospective investigations are being carried out regarding any benefits in terms of reducing the time required to manage claims and saving on the amounts liquidated.

Despite these limitations, this study represents one of the first attempts to identify a standardized approach for the management of HAI claims that can be used in all hospital settings. Although this study shares the limitations of singlecenter studies, it has the enormous advantage of relying on a relatively large case series based on direct analysis of medi- cal records and not on administrative data such as those used in major epidemiological investigations. Furthermore, the proposed integrated approach represents a reasonable and cost-effective method for managing claims arising from Hospital Acquired Infections.

Finally, the simplicity of the proposed method makes it possible to apply it even in less advanced hospital settings. Moreover, the implementation and adaptation of the tools described to the specificities of different cases of medical liability offer numerous research perspectives for the application of similar systems to the general claims.

\section{CONCLUSION}

Hospital Acquired Infections have a critical impact on health systems representing a major issue in terms of morbidity and mortality, lengthening of hospital stay, direct and indirect costs.

Given the complexity and severity of HAIs emerged from this study and several other scientific contributions, the necessity to implement prevention strategies and quality of care is evident. In this regard, specific epidemiological surveillance measures and monitoring of care pathways appear to be of fundamental importance to drastically reduce the incidence of nosocomial infections and consequently the morbidity and mortality associated with them.

In view of the preventability of most nosocomial infections, a better understanding of the clinical and economic impact of the infectious complications related to care may undoubtedly provide useful indications for the implementation of care practices and the improvement of patient safety. In this regard, the study of HAI claims can be functional for the identification of suboptimal steps in the chain of care able to threaten the patient's safety as well as the planning of any corrective measures to the preventive measures in use [69-74].

The proposed approach allows a productive analysis of the internal processes, providing fundamental data for the refinement of the preventive strategies and for the management of the resources through the expenditure forecasts [75]. Furthermore, the methodology described has proved to be fundamental in the development of strategies aimed at rationalizing economic resources and corporate policies in the field of quality of care.

The integrated management of claims arising from Healthcare Associated Infections using the method described in this study requires the joint work of different specialists in the field presupposing an indispensable multidisciplinary approach for the solution of problems such as those related to nosocomial infections.

In conclusion, it is possible to affirm that understanding data on the HAI claims represents a fundamental prerequisite for determining the risk of loss and technical estimates. Data exploration must be based on the rational interpretation of trends affecting claims. [76] Only in this way exploration can help analysts to select the most appropriate predictive methods and to interpret the results obtained. Therefore, pending further studies for method consolidation and trend analysis, ALEA iacta est. 


\section{LIST OF ABBREVIATIONS}

$$
\begin{aligned}
& \text { ALEA }=\text { Advanced Loss Eventuality Assessment } \\
& \text { CR-BSI }=\text { Catheter-Related Bloodstream Infections } \\
& \text { HAI = Healthcare-Associated Infections } \\
& \text { ICPS }=\text { International Classification for Patient Safety } \\
& \text { VAP }=\text { Ventilator-Associated Pneumonia }
\end{aligned}
$$

\section{ETHICS APPROVAL AND CONSENT TO PARTICI- PATE}

Not applicable.

\section{HUMAN AND ANIMAL RIGHTS}

No Animals/Humans were used for studies that are base of this research.

\section{CONSENT FOR PUBLICATION}

Not applicable.

\section{AVAILABILITY OF DATA AND MATERIALS}

Not applicable.

\section{FUNDING}

None.

\section{CONFLICT OF INTEREST}

The authors declare no conflict of interest, financial or otherwise.

\section{ACKNOWLEDGEMENTS}

Declared none.

\section{AUTHORS' ROLES}

All Authors contributed equally to manuscript drafting and critical discussion and approved the final version.

\section{REFERENCES}

[1] The National Quality Forum. Safe practices for better healthcare 2010 update: a consensus report. National Quality Forum, 2010.

[2] Pawlson, L.G.; O'Kane, M.E. Malpractice prevention, patient safety, and quality of care: a critical linkage. Am. J. Manag. Care, 2004, 10(4), 281-284

[3] Mirzoev, T.; Kane, S. Key strategies to improve systems for managing patient complaints within health facilities - what can we learn from the existing literature? Glob. Health Action, 2018, 11(1), 1458938.

[4] Hsieh, S.Y. The use of patient complaints to drive quality improvement: an exploratory study in Taiwan. Health Serv. Manage. Res., 2010, 23(1), 5-11.

[5] Bates, D.W.; Larizgoitia, I.; Prasopa-Plaizier, N.; Jha, A.K.; Research Priority Setting Working Group of the WHO World Alliance for Patient Safety. Global priorities for patient safety research. $B M J, 2009,338$, b1775.

[6] Burke, J.P. Infection control - a problem for patient safety. N. Engl. J. Med., 2003, 348(7), 651-656.

[7] Ducel, G.; Fabry, J.; Nicolle, L.; Girard, R.; Perraud, M.; Prüss, A. Savey, A.; Tikhomirov, E.; Thuriaux, M.; Vanhems, P. Prevention of hospital-acquired infections: a practical guide. World Health Organization, 2002.
[8] Gastmeier, P.; Geffers, C.; Brandt, C.; Zuschneid, I.; Sohr, D.; Schwab, F.; Behnke, M.; Daschner, F.; Rüden, H. Effectiveness of a nationwide nosocomial infection surveillance system for reducing nosocomial infections. J. Hosp. Infect., 2006, 64(1), 16-22.

[9] European Centre for Disease Prevention and Control. Point prevalence survey of healthcare-associated infections and antimicrobial use in European acute care hospitals. European Centre for Disease Prevention and Control, 2013.

[10] European Centre for Disease Prevention and Control. Annual Epidemiological Report 2012. Reporting on 2010 surveillance data and 2011 epidemic intelligence data. European Centre for Disease Prevention and Control, 2013.

[11] Kohn, L.T.; Corrigan, J.M.; Donaldson, M.S.; Institute of Medicine (US) Committee on Quality of Health Care in America. To Err Is Human: Building a Safer Health System. National Academies Press: Washington, 2000.

[12] Al-Tawfiq, J.A.; Tambyah, P.A. Healthcare associated infections (HAI) perspectives. J. Infect. Public Health, 2014, 7(4), 339-344.

[13] Jha, A.K.; Chan, D.C.; Ridgway, A.B.; Franz, C.; Bates, D.W. Improving safety and eliminating redundant tests: cutting costs in US hospitals. Health Aff. (Millwood), 2009, 28(5), 1475-1484.

[14] National Quality Forum. Safe practices for better healthcare: 2010 update.

http://www.qualityforum.org/Projects/Safe_Practices_2010.aspx (Accessed June 14, 2018).

[15] Truskett, P. Should we view patient complaints as a resource? ANZ J. Surg., 2012, 82(10), 667.

[16] Jonsson, P.M.; Øvretveit, J. Patient claims and complaints data for improving patient safety. Int. J. Health Care Qual. Assur., 2008 $21(1), 60-74$

[17] Øvretveit, J.; Klazinga, N. Guidance on developing quality and safety strategies with a health system approach. World Health Organization, 2008.

[18] Pichert, J.W.; Moore, I.N.; Karrass, J.; Jay, J.S.; Westlake, M.W.; Catron, T.F.; Hickson, G.B. An intervention model that promotes accountability: peer messengers and patient/family complaints. $J$. Comm. J. Qual. Patient Saf., 2013, 39(10), 435-446.

[19] Sun, X.; Faunce, T. Decision-analytical modelling in health-care economic evaluations. Eur. J. Health Econ., 2008, 9(4), 313-323.

[20] Lisboa, T.; Rello, J. Towards zero rate in healthcare-associated infections: one size shall not fit all. Crit. Care, 2013, 17(3), 139.

[21] Harbarth, S.; Sax, H.; Gastmeier, P. The preventable proportion of nosocomial infections: an overview of published reports. J. Hosp. Infect., 2003, 54(4), 258-266.

[22] Umscheid, C.A.; Mitchell, M.D.; Doshi, J.A.; Agarwal, R.; Williams, K.; Brennan, P.J. Estimating the proportion of healthcareassociated infections that are reasonably preventable and the related mortality and costs. Infect. Control Hosp. Epidemiol., 2011, 32(2), 101-114.

[23] Safdar, N.; Anderson, D.J.; Braun, B.I.; Carling, P.; Cohen, S Donskey, C.; Drees, M.; Harris, A.; Henderson, D.K.; Huang, S.S.; Juthani-Mehta, M.; Lautenbach, E.; Linkin, D.R.; Meddings, J.; Miller, L.G.; Milstone, A.; Morgan, D.; Sengupta, S.; Varman, M.; Yokoe, D.; Zerr, D.M.; Research Committee of the Society for Healthcare Epidemiology of America. Infect. Control Hosp. Epidemiol., 2014, 35(5), 480-493.

[24] Clancy, C.M. Getting to zero: our effort to eliminate infections nationwide. J. Nurs. Care Qual., 2010, 25(3), 189-192.

[25] Agency for Healthcare Research and Quality (AHRQ). Fact Sheet AHRQ's Efforts to Prevent and Reduce Healthcare-Associated Infections.

https://archive.ahrq.gov/research/findings/factsheets/errorssafety/haiflyer/haiflyer.pdf (Accessed June 12, 2018)

[26] Boncagni, F.; Francolini, R.; Nataloni, S.; Skrami, E.; Gesuita, R.; Donati, A.; Pelaia, P. Epidemiology and clinical outcome of healthcare-associated infections: a 4- year experience of an Italian ICU. Minerva Anestesiol., 2015, 81(7), 765-775.

[27] Burgmann, H.; Hiesmayr, J.M.; Savey, A.; Bauer, P.; Metnitz, B.; Metnitz, P.G. Impact of nosocomial infections on clinical outcome and resource consumption in critically ill patients. Intensive Care Med., 2010, 36(9) 1597-1601.

[28] Thursky, K.1.; Lingaratnam, S.; Jayarajan, J.; Haeusler, G.M.; The, B.; Tew, M.; Venn, G.; Hiong, A.; Brown, C.; Leung, V.; Worth, L.J.; Dalziel, K.; Slavin, M.A. Implementation of a whole of hospital sepsis clinical pathway in a cancer hospital: impact on sepsis 
management, outcomes and costs. BMJ Open Qual., 2018, 7(3), e000355.

[29] Agodi, A.; Auxilia, F.; Barchitta, M.; Brusaferro, S.; D'Alessandro, D.; Grillo, O.C.; Montagna, M.T.; Pasquarella, C.; Righi, E.; Tardivo, S.; Torregrossa, V.; Mura, I.; GISIO-SITI. Trends, risk factors and outcomes of healthcare-associated infections within the Italian network SPIN-UTI. J. Hosp. Infect., 2013, 84(1), 52-58.

[30] Siempos, I.I.; Kopterides, P.; Tsangaris, I.; Dimopoulou, I.; Armaganidis, A.E. Impact of catheter-related bloodstream infections on the mortality of critically ill patients: a meta-analysis. Crit. Care Med., 2009, 37(7), 2283-2289.

[31] Bekaert, M.; Timsit, J.F.; Vansteelandt, S.; Depuydt, P.; Vésin, A.; Garrouste-Orgeas, M.; Decruyenaere, J.; Clec'h, C.; Azoulay, E.; Benoit, D.; Outcomerea Study Group. Attributable mortality of ventilator-associated pneumonia: a reappraisal using causal analysis. Am. J. Respir. Crit. Care Med., 2011, 184(10), 1133-1139.

[32] Eber, M.R.; Laxminarayan, R.; Perencevich, E.N.; Malani, A. Clinical and economic outcomes attributable to health careassociated sepsis and pneumonia. Arch. Intern. Med., 2010, 170(4), 347-353.

[33] Stone, P.W. Economic burden of healthcare-associated infections: an American perspective. Expert Rev. Pharmacoecon. Outcomes Res., 2009, 9(5), 417-422.

[34] Stone, P.W.; Braccia, D.; Larson, E. Systematic review of economic analyses of health care-associated infections. Am. J. Infect. Control, 2005, 33(9), 501-509.

[35] Zimlichman, E.; Henderson, D.; Tamir, O.; Franz, C.; Song, P.; Yamin, C.K.; Keohane, C.; Denham, C.R.; Bates, D.W. Health care-associated infections: a meta-analysis of costs and financial impact on the US health care system. JAMA Intern. Med., 2013, 173(22), 2039-2046

[36] Klevens, R.M.; Edwards, J.R.; Richards, C.L. Jr; Horan, T.C.; Gaynes, R.P.; Pollock, D.A.; Cardo, D.M. Estimating health careassociated infections and deaths in US hospitals, 2002. Public Health Rep., 2007, 122(2), 160-166.

[37] European Centre for Disease Prevention and Control. Annual epidemiological report on communicable diseases in Europe 2008. Report on the state of communicable diseases in the EU and EEA/EETA countries. European Centre for Disease Prevention and Control, 2008.

[38] Vincent, J.L. Nosocomial infections in adult intensive-care units. Lancet, 2003, 361(9374), 2068-2077.

[39] Vincent, J.L.; Bihari, D.J.; Suter, P.M.; Bruining, H.A.; White, J.; Nicolas-Chanoin, M.H.; Wolff, M.; Spencer, R.C.; Hemmer, M. The prevalence of nosocomial infection in intensive care units in Europe. Results of the European Prevalence of Infection in Intensive Care (EPIC) study. EPIC International Advisory Committee. $J A M A$, 1995, 274(8) 639-644.

[40] Hugonnet, S.; Eggimann, P.; Borst, F.; Maricot, P.; Chevrolet, J.C.; Pittet, D. Impact of ventilator-associated pneumonia on resource utilization and patient outcome. Infect. Control Hosp. Epidemiol., 2004, 25(12), 1090-1096.

[41] Safdar, N.; Dezfulian, C.; Collard, H.R.; Saint, S. Clinical and economic consequences of ventilator-associated pneumonia: a systematic review. Crit. Care Med., 2005, 33(10), 2184-2193.

[42] O'Grady, N.P.; Alexander, M.; Burns, L.A.; Dellinger, E.P.; Garland, J.; Heard, S.O.; Lipsett, P.A.; Masur, H.; Mermel, L.A.; Pearson, M.L.; Raad, I.I.; Randolph, A.G.; Rupp, M.E.; Saint, S.; Healthcare Infection Control Practices Advisory Committee (HICPAC). Guidelines for the prevention of intravascular catheterrelated infections. Clin. Infect. Dis., 2011, 52(9), e162-93.

[43] Bishop, T.F.; Ryan, A.M.; Casalino, L.P. Paid malpractice claims for adverse events in inpatient and outpatient settings. JAMA, 2011, 305(23), 2427-2431.

[44] Bonetti, M.; Cirillo, P.; Musile Tanzi, P.; Trinchero, E. An Analysis of the Number of Medical Malpractice Claims and Their Amounts. PLoS One, 2016, 11(4), e0153362.

[45] McKibben, L.; Horan, T.; Tokars, J.I.; Fowler, G.; Cardo, D.M.; Pearson, M.L.; Brennan, P.J.; Healthcare Infection Control Practices Advisory Committee. Guidance on public reporting of healthcare-associated infections: recommendations of the Healthcare Infection Control Practices Advisory Committee. Infect. Control Hosp. Epidemiol., 2005, 26(6), 580-587.

[46] Haustein, T.; Gastmeier, P.; Holmes, A.; Lucet, J.C.; Shannon, R.P.; Pittet, D.; Harbarth, S. Use of benchmarking and public re- porting for infection control in four high-income countries. Lancet Infect. Dis., 2011, 11(6), 471-481.

[47] Javetz, R.; Stern, Z. Patients' complaints as a management tool for continuous quality improvement. J. Manag. Med., 1996, 10(3), 3948.

[48] Hsieh, S.Y. Using complaints to enhance quality improvement: developing an analytical tool. Int. J. Health Care Qual. Assur., 2012, 25(5), 453-461.

[49] Greve, P.A. Jr. Anticipating and controlling rising malpractice insurance costs. Healthc. Financ. Manage., 2002, 56(5), 50-55.

[50] Yokoe, D.S.; Anderson, D.J.; Berenholtz, S.M.; Calfee, D.P.; Dubberke, E.R.; Ellingson, K.D.; Gerding, D.N.; Haas, J.P.; Kaye, K.S.; Klompas, M.; Lo, E.; Marschall, J.; Mermel, L.A.; Nicolle, L.E.; Salgado, C.D.; Bryant, K.; Classen, D.; Crist, K.; Deloney, V.M.; Fishman, N.O.; Foster, N.; Goldmann, D.A.; Humphreys, E.; Jernigan, J.A.; Padberg, J.; Perl, T.M.; Podgorny, K.; Septimus, E.J.; VanAmringe, M.; Weaver, T.; Weinstein, R.A.; Wise, R.; Maragakis, L.L.; Society for Healthcare Epidemiology of America (SHEA). A compendium of strategies to prevent healthcareassociated infections in acute care hospitals: 2014 updates. Infect. Control. Hosp. Epidemiol., 2014, 35(8), 967-977.

[51] Ranji, S.R.; Shetty, K.; Posley, K.A.; Lewis, R.; Sundaram, V.; Galvin, C.M.; Winston, L.G. Prevention of Healthcare-Associated Infections. Agency for Healthcare Research and Quality, 2007, AHRQ Publication No. 04(07)-0051-6.

[52] Scott, D.R. The direct medical costs of healthcare-associated infections in US hospitals and the benefits of prevention. https://www.cdc.gov/hai/pdfs/hai/scott_costpaper.pdf (Accessed June 7, 2018).

[53] Magill, S.S.; Edwards, J.R.; Bamberg, W.; Beldavs, Z.G.; Dumyati, G.; Kainer, M.A.; Lynfield, R.; Maloney, M.; McAllister-Hollod, L.; Nadle, J.; Ray, S.M.; Thompson, D.L.; Wilson, L.E.; Fridkin, S.K.; Emerging Infections Program Healthcare-Associated Infections and Antimicrobial Use Prevalence Survey Team. Multistate point-prevalence survey of health care-associated infections. $N$. Engl. J. Med., 2014, 370(13), 1198-1208.

[54] van der Wal, G.; Lens, P. Handling complaints in hospitals. Health Policy, 1994, 31(1) 17-27.

[55] Cowan, J.; Anthony, S. Problems with complaint handling: expectations and outcomes. Clin. Governance: Int. J., 2008, 13(2), 164168.

[56] van Bunnik, B.A.; Ciccolini, M.; Gibbons, C.L.; Edwards, G.; Fitzgerald, R.; McAdam, P.R.; Ward, M.J.; Laurenson, I.F.; Woolhouse, M.E. Efficient national surveillance for health-careassociated infections. BMC Public Health, 2015, 15, 832.

[57] Mitchell, B.G.; Russo, P.L. Preventing healthcare-associated infections: the role of surveillance. Nurs. Stand., 2015, 29(23), 52-58.

[58] Gastmeier, P. Surveillance of nosocomial infections. Ther Umsch., 2004, 61(3), 204-209.

[59] Centers for Disease Control and Prevention. Monitoring hospitalacquired infections to promote patient safety - United States, 19901999. MMWR Morb. Mortal. Wkly. Rep., 2000, 49(8), 149-153.

[60] Platt, R.; Yokoe, D.S.; Sands, K.E. Automated methods for surveillance of surgical site infections. Emerg. Infect. Dis., 2001, 7(2), 212-216.

[61] Weigelt, J.A.; Dryer, D.; Haley, R.W. The necessity and efficiency of wound surveillance after discharge. Arch Surg, 1992, 127(1). 7781.

[62] Samore, M.H.; Evans, R.S.; Lassen, A.; Gould, P.; Lloyd, J.; Gardner, R.M.; Abouzelof, R.; Taylor, C.; Woodbury, D.A.; Willy, M.; Bright, R.A. Surveillance of medical device-related hazards and adverse events in hospitalized patients. JAMA, 2004, 291(3), 325334.

[63] O'Neill, E.; Humphreys, H. Use of surveillance data for prevention of healthcare-associated infection: risk adjustment and reporting dilemmas. Curr. Opin. Infect. Dis., 2009, 22(4), 359-363.

[64] Emori, T.G.; Culver, D.H.; Horan, T.C.; Jarvis W.R.; White, J.W.; Olson, D.R.; Banerjee, S.; Edwards, J.R.; Martone, W.J.; Gaynes, R.P.; Hughes, J.M. National nosocomial infections surveillance system (NNIS): description of surveillance methods. Am. J. Infect. Control, 1991, 19(1), 19-35.

[65] Suetens, C.; Morales, I.; Savey, A.; Palomar, M.; Hiesmayr, M.; Lepape, A.; Gastmeier, P.; Schmit, J.C..; Valinteliene, R.; Fabry, J. European surveillance of ICU-acquired infections (HELICS-ICU): methods and main results. J. Hosp. Infect., 2007, 65(2), 171-173. 
[66] Wilson, J.; Ramboer, I.; Suetens, C. Hospitals in Europe Link for Infection Control through Surveillance (HELICS). Inter-country comparison of rates of surgical site infection - Opportunities and limitations. J. Hosp. Infect., 2007, 65(2), 165-170.

[67] Mello, M.M.; Studdert, D.M. Building a National Surveillance System for Malpractice Claims. Health Serv Res, 2016, 51(3), 2642-2648.

[68] Bismark, M.M.; Studdert, D.M. Realising the research power of complaints data. N. Z. Med. J., 2010, 123(1314), 12-17.

[69] Mu, Y.; Edwards, J.R.; Horan, T.C.; Berrios-Torres, S.I.; Fridkin, S.K. Improving risk-adjusted measures of surgical site infection for the national healthcare safety network. Infect. Control. Hosp. Epidemiol, 2011, 32(10):970-986.

[70] Järvelin, J.; Häkkinen, U. Can patient injury claims be utilised as a quality indicator? Health Policy, 2012, 104(2), 155-162.

[71] Smith, P.C.; Mossialos, E.; Leatherman, S.; Papanicolas, I. Performance measurement for health system improvement - Experiences, challenges and prospects; Cambridge University Press: Cambridge, 2009.

[72] Jonsson, P.M.; Øvretveit, J. Patient claims and complaints data for improving patient safety. Int. J. Health Care Qual. Assur., 2008, 2l(1), 60-74.

[73] Levin, C.M.; Hopkins, J. Creating a patient complaint capture and resolution process to incorporate best practices for patient-centered representation. Jt. Comm. J. Qual. Patient Saf., 2014, 40(11), 484492.

[74] Hsieh, S.Y. A system for using patient complaints as a trigger to improve quality. Qual. Manag. Health Care, 2011, 20(4), 343-355.

[75] Simmons, J.C. How root-cause analysis can improve patient safety. Qual. Lett. Healthc. Lead., 2001, 13(10), 2-12.

[76] Landrigan, C.P.; Parry, G.J.; Bones, C.B.; Hackbarth, A.D.; Goldmann, D.A.; Sharek, P.J. Temporal trends in rates of patient harm resulting from medical care. N. Engl. J. Med., 2010, 363(22), 21242134. 